\title{
Will the real Golgi please stand up
}

\author{
It was discovered more than a century ago, but cell biologists are still \\ debating whether the Golgi complex is an autonomous entity. \\ Erika Check profiles an organelle in identity crisis.
}

$\mathrm{E}$ ver since the Golgi complex was first described in 1898, this embattled cellular organelle has struggled to secure an identity for itself. Using his Nobelwinning method of staining cells with silver salts, the Italian biologist Camillo Golgi spotted that neurons contain a stack of flattened, membrane-bound sacs. But although the same structure is found in all nucleated cells, from humans to amoebae, naysayers argued for decades that it was merely an artefact of Golgi's staining technique.

In the 1950s the electron microscope finally proved that the Golgi was no artefact. And the organelle gained further legitimacy when researchers revealed its crucial function: processing and packaging proteins for export from the cell.

But now, the Golgi's integrity is again in doubt. At issue is the question of whether it is a truly independent organelle, persisting through cell division in skeletal form and being rebuilt from this template. One camp of cell biologists, led by Graham Warren at Yale University in New Haven, Connecticut, subscribes to this view. But others, championed by Jennifer Lippincott-Schwartz at the National Institute of Child Health and Human Development (NICHD) in Bethesda, Maryland, argue that the Golgi is just a fleeting aggregation of proteins and lipid membrane that constantly assembles and disassembles.

\section{Dynamic cells}

The Golgi question plays into a wider debate about how cells are built. The classic view is that cells make new parts by assembling them on static frameworks, rather as a skyscraper is built around a steel scaffold. But some biologists argue that many intracellular structures are constantly forming and disappearing in a flexible process that does not depend on underlying templates - like the dynamic equilibrium between condensation and evaporation in a billowing cloud. "This idea of dynamic self-organization is becoming more and more popular in many areas of cell biology," says Ben Glick, who studies the Golgi complex at the University of Chicago.

The Golgi's current identity crisis stems from the late 1980s, when Lippincott-
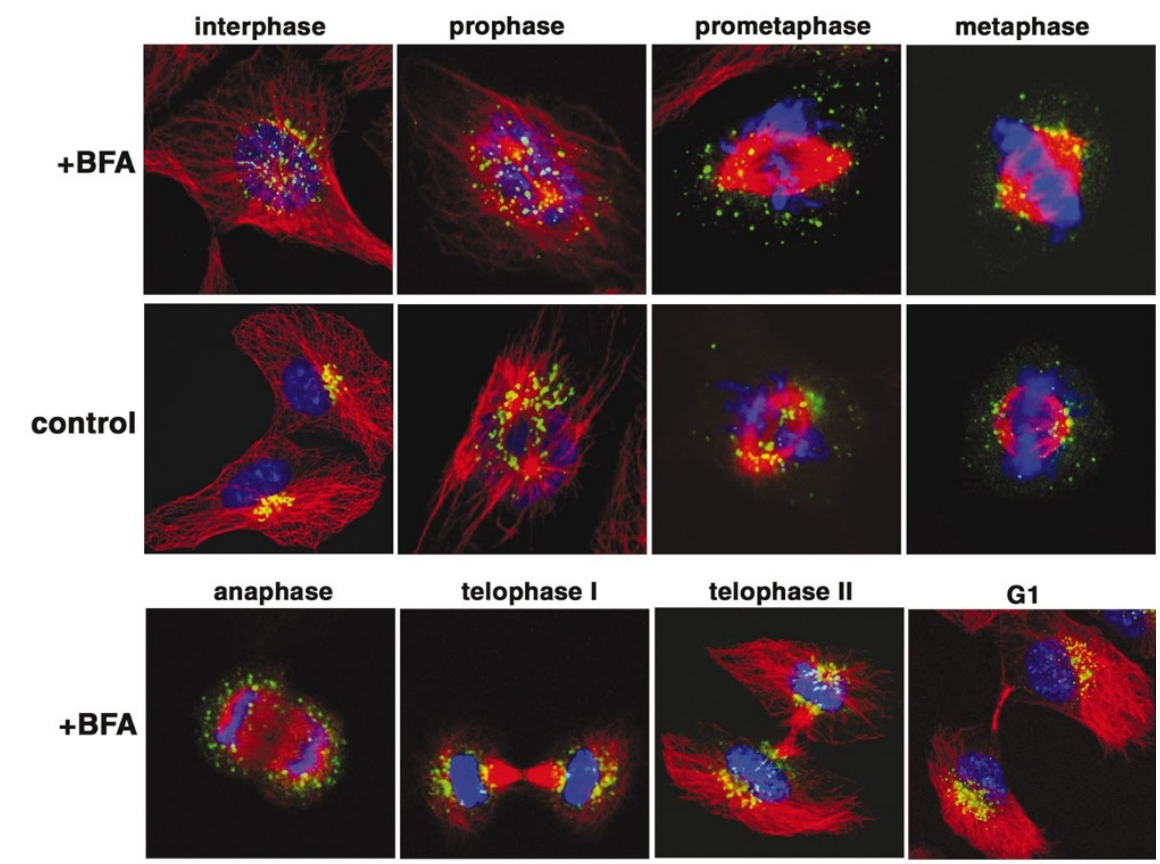

telophase I

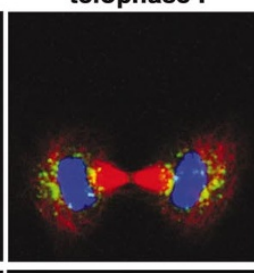

telophase II

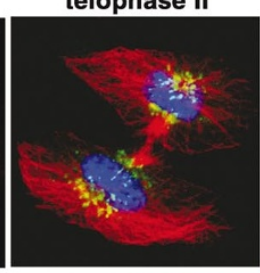

G1
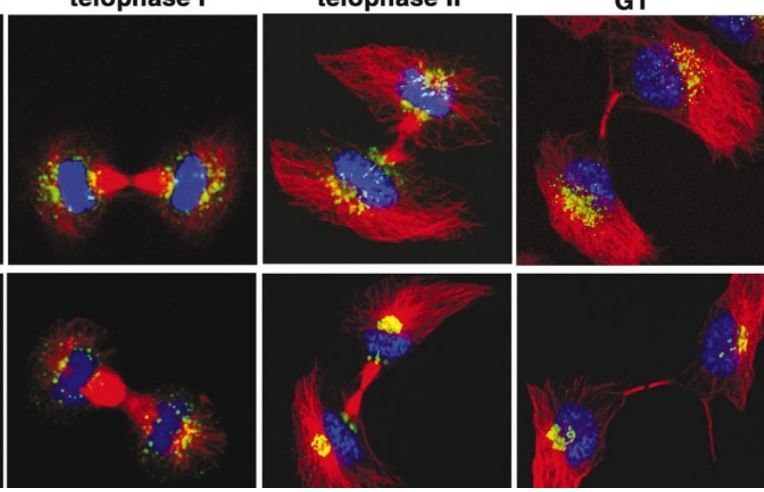

Graham Warren thinks that 'matrix' proteins (stained green, above) persist throughout cell division and act as templates for the Golgi's reassembly.

Schwartz was working in Richard Klausner's lab at the NICHD as a postdoc. She gave cells a shot of brefeldin A, an antibiotic from fungi that blocks the transport of proteins from the endoplasmic reticulum (ER) to the Golgi. The ER is another complex of intracellular membranes; it receives proteins directly from proteinbuilding ribosomes attached to its surface. When transport from the ER to the Golgi was blocked, the Golgi disintegrated, and proteins associated with its membranes were rapidly redistributed to the ER. And when Lippincott-Schwartz removed the antibiotic, the Golgi reappeared ${ }^{1}$.

To Lippincott-Schwartz and her colleagues, this indicated that the Golgi is constantly recycled to and from the ER in a dynamic equilibrium ${ }^{2}$. "This was the first crack in the theory that the Golgi is a stable, pre-existing entity," she says.

More recently, her group studied what happens to the Golgi during cell division, when its structure temporarily breaks down. By labelling Golgi proteins with green fluorescent protein, the researchers showed that these proteins fled to the ER. After cell division was complete, the ER spat out the Golgi proteins, and the structure rebuilt itself. But rebuilding could be prevented by expressing a mutant version of a gene called Sar1, which - like brefeldin A — blocks the transport of proteins from the $\mathrm{ER}^{3}$.

In the meantime, Warren and his colleagues had been looking more closely at cells disturbed by brefeldin A. Like LippincottSchwartz's team, they found that a dose of the antibiotic caused Golgi proteins to migrate to the ER. But not all of them. While working at the Imperial Cancer Research Fund's laboratories in London in the mid-1990s, Warren 
showed that one protein, called GM130, stayed behind". "This gave us the idea that such proteins might be the structure underlying the framework of the Golgi," says Warren. "Taking an extreme view, you can argue that this is the Golgi apparatus itself."

Although GM130 and other matrix proteins disperse through the cytoplasm when cells are dosed with brefeldin A, Warren has found that they still form Golgilike structures in cells expressing a mutant Sar1 gene, in which other Golgi proteins the enzymes that process proteins destined for export from the cell - become confined to the $\mathrm{ER}^{5}$.

\section{Tracking the template}

In February this year, Warren and his colleagues reported on their efforts to track the matrix proteins during cell division. Again using brefeldin A or mutant Sar1 to confine Golgi enzymes to the ER, they showed that matrix proteins were partitioned into the daughter cells in a manner reminiscent of the entire organelle. Using microscopic magnetic beads labelled with a fluorescent antibody that captures GP130, the researchers also found that this matrix protein remained distinct from the $\mathrm{ER}^{6}$.

This convinces Warren that matrix proteins are the template from which the Golgi is rebuilt after cell division. "Our take on this is that the Golgi is an independent organelle responsible for its own partitioning and the endoplasmic reticulum doesn't play a part in this," he says.

Lippincott-Schwartz prets the results differently. She accepts that the matrix proteins stay separate from the main body of the ER in cells treated with brefeldin A. But she argues that the matrix proteins travel to a specialized part of the ER called its 'exit site', from which membranes pinch off and carry their cargo of proteins to the Golgi. It is this portion of the ER, LippincottSchwartz believes, that directs the Golgi's reassembly.

She bases this claim on studies of dividing cells treated with brefeldin A, in which her team labelled the ER exit sites and matrix proteins using different fluorescent dyes. The two labels overlapped, and the labelled matrix proteins moved away from the Golgi before the other proteins in the organelle. This contradicts the idea that the matrix proteins stay behind after the Golgi unravels, Lippincott-Schwartz argues. Her team has also used a different mutant of Sar1 that prevents the production of ER exit sites, and found that the Golgi completely fails to reform after treatment with brefeldin $\mathrm{A}^{7}$.

For now, the debate over whether the Golgi is an autonomous entity remains unresolved. Although Lippincott-Schwartz's

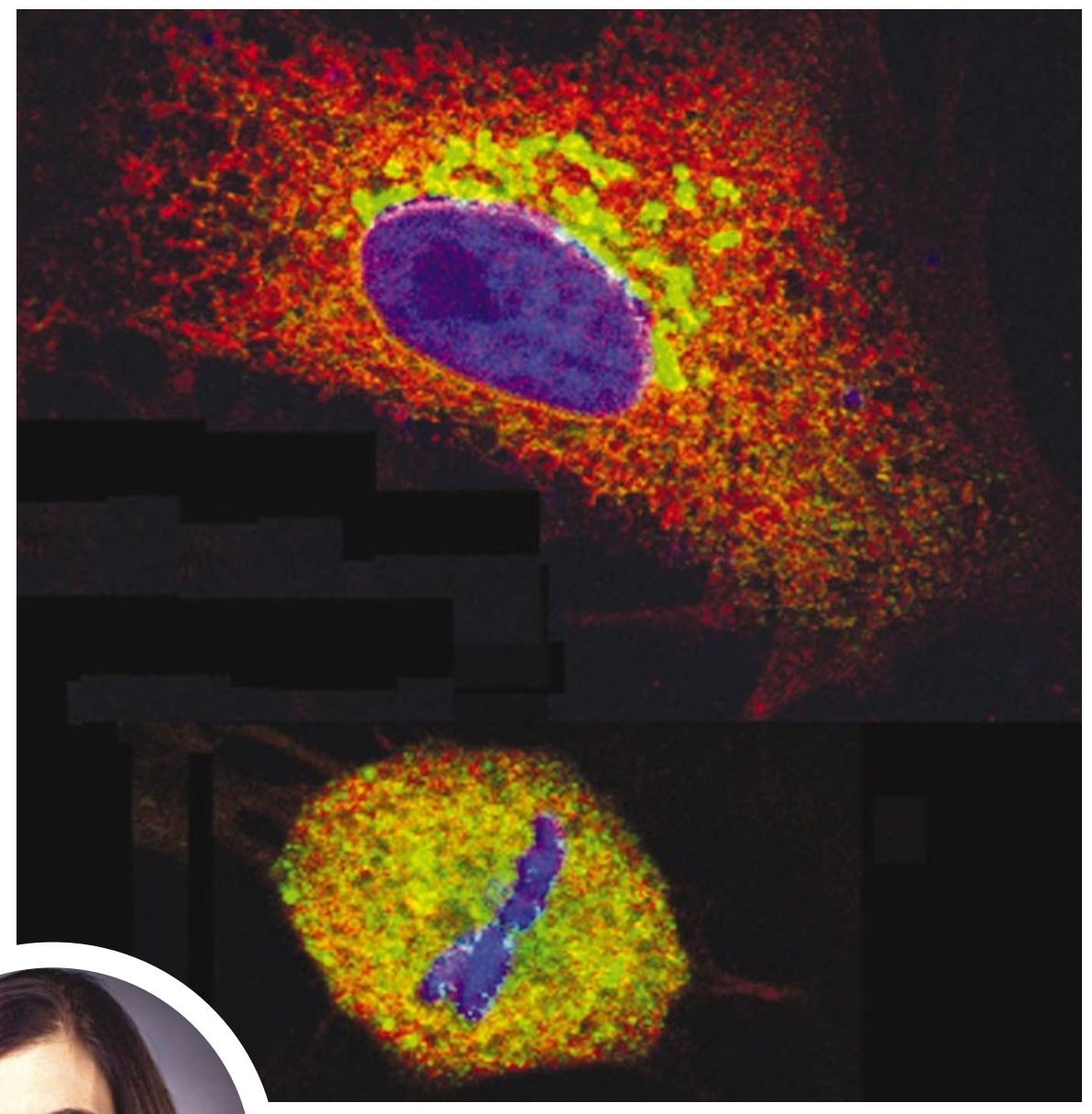

Mixing of Golgi (green) and endoplasmic reticulum (red) before a cell divides (bottom) makes Jennifer Lippincott-Schwartz question the Golgi's autonomy.

results suggest that the organelle is much more mutable than was once thought, other cell biologists say it is possible that we just do not know enough about the Golgi to be sure about what is going on when it reassembles. Perhaps the matrix proteins that direct the process are yet to be discovered, they suggest. "People are trying to define the Golgi based on four or five markers," observes Vivek Malhotra of the University of California, San Diego. "What if there is a complex of proteins we don't know about, and that is serving as a sort of nucleation site?"

\section{Self-sufficiency}

In addition, say some experts, we need to find out more about what happens to the Golgi during and after cell division, in the absence of any experimental disruption. If the Golgi reforms very quickly after cells divide, it would support the idea that it is being rebuilt from a residual template, rather than being recycled from the ER and assembling completely from scratch.

But the idea of dynamic self-organization has attractions that extend well beyond the Golgi. The reassembly of the nucleus from pre-existing skeletal structures might be the exception rather than the rule. If cellular structures were mostly assembled through dynamic and self-organizing protein interactions, it would help explain cells' tremendous flexibility in responding to changes in their environment.

"Self-organization makes a lot of sense when you think about what a cell has to do," says Tom Misteli, a cell biologist at the National Cancer Institute in Bethesda. "It allows a cell to be very stable, but on the other hand something terrible can happen to a cell at any moment, and it has to be able to respond to that."

Seen in this light, the Golgi's identity crisis seems less neurotic than noble. Far from being an isolated search for legitimacy, its resolution might provide fundamental insights into the way cells are built.

Erika Check is Nature's Washington biomedical

\section{correspondent.}

1. Lippincott-Schwartz, J., Yuan, L. C., Bonifacino, J. S. \& Klausner R. D. Cell 56, 801-813 (1989).

2. Klausner, R. D., Donaldson, R. G. \& Lippincott-Schwartz, J. J. Cell Biol. 116, 1071-1081 (1992).

3. Zaal, K. J. M. et al. Cell 99, 589-601 (1999).

4. Nakamura, N. et al. J. Cell Biol. 131, 1715-1726 (1995)

5. Seemann, J., Jokitalo, E., Pypaert, M. \& Warren, G. Nature 407, 1022-1026 (2000).

6. Seemann, J., Pypaert, M., Taguchi, T., Malsam, J. \& Warren, G. Science 295, 848-851 (2002).

7. Ward, T. H., Polishchuk, R., Caplan, S., Hirschberg, K. \& Lippincott-Schwartz, J. J. Cell Biol. 155, 557-570 (2001). 